\title{
Clinical Presentation and Microarray Analysis of Peruvian Children with Atypical Development and/or Aberrant Behavior
}

\author{
Merlin G. Butler, ${ }^{1,2}$ Kelly Usrey, ${ }^{1}$ Jennifer L. Roberts, \\ Stephen R. Schroeder, ${ }^{3}$ and Ann M. Manzardo ${ }^{1}$ \\ ${ }^{1}$ Department of Psychiatry and Behavioral Sciences, The University of Kansas Medical Center, \\ 3901 Rainbow Boulevard, MS 4015, Kansas City, KS 66160, USA \\ ${ }^{2}$ Kansas University Center on Development Disabilities, Schiefelbusch Institute for Life Span Institute, \\ The University of Kansas, Lawrence, KS 66045, USA \\ ${ }^{3}$ Department of Pediatrics, The University of Kansas Medical Center, Kansas City, KS 66160, USA
}

Correspondence should be addressed to Merlin G. Butler; mbutler4@kumc.edu

Received 17 July 2014; Accepted 1 October 2014; Published 20 October 2014

Academic Editor: Francine Durocher

Copyright (C) 2014 Merlin G. Butler et al. This is an open access article distributed under the Creative Commons Attribution License, which permits unrestricted use, distribution, and reproduction in any medium, provided the original work is properly cited.

\begin{abstract}
We report our experience with high resolution microarray analysis in infants and young children with developmental disability and/or aberrant behavior enrolled at the Centro Ann Sullivan del Peru in Lima, Peru, a low income country. Buccal cells were collected with cotton swabs from 233 participants for later DNA isolation and identification of copy number variation (deletions/duplications) and regions of homozygosity $(\mathrm{ROH})$ for estimating consanguinity status in 15 infants and young children (12 males, 3 females; mean age $\pm S D=28.1 \mathrm{~m} \pm 7.9 \mathrm{~m}$; age range $14 \mathrm{~m}-41 \mathrm{~m}$ ) randomly selected for microarray analysis. An adequate DNA yield was found in about one-half of the enrolled participants. Ten participants showed deletions or duplications containing candidate genes reported to impact behavior or cognitive development. Five children had ROHs which could have harbored recessive gene alleles contributing to their clinical presentation. The coefficient of inbreeding was calculated and three participants showed first-second cousin relationships, indicating consanguinity. Our preliminary study showed that DNA isolated from buccal cells using cotton swabs was suboptimal, but yet in a subset of participants the yield was adequate for high resolution microarray analysis and several genes were found that impact development and behavior and ROHs identified to determine consanguinity status.
\end{abstract}

\section{Introduction}

Research involving early recognition of developmental/intellectual disabilities with or without aberrant behaviors such as self-injury, aggression, stereotypic patterns, and autism is on the increase but the causation is poorly understood. Advances in genetic testing now allow for the identification of genetic disturbances including chromosomal abnormalities and gene mutations or variants [1]. Most recently, the rise of chromosomal microarrays or comparative genomic hybridization arrays $(\mathrm{aCGH})$ is increasingly helpful for determination of copy number variants (CNV) by identifying subtle DNA deletions and duplications that contribute significantly to genomic variation (e.g., $21 \%$ of consecutive patients with autism or intellectual disability presenting for genetic services at an academic setting) [2].

Single nucleotide polymorphisms (SNPs) are also helpful in detecting regions of homozygosity [large genomic regions without SNPs which may indicate gene alleles identical by descent from a common ancestor with evidence of consanguinity or due to areas of loss of heterozygosity (e.g., uniparental maternal disomy of chromosome 15 in which both chromosome 15s are inherited from the mother causing Prader-Willi syndrome, a classical genetic disorder due to errors in genomic imprinting)] [3-5]. Consanguinity is a common occurrence in some cultures and offspring of consanguineous or related couples sharing gene alleles are more likely to inherit mutant alleles for disease, particularly 
autosomal recessive conditions. The working definition of consanguinity is a union between individuals whom are biologically related as second cousins or closer [6]. Offspring of consanguineous parents also have increased risks for birth defects and multifactorial disorders [7]. The level of consanguinity in an individual can be empirically measured and quantified as the coefficient of inbreeding $(F)$ based upon the level of homozygosity in the genome and assessed using SNP data from microarray analysis [3]. Couples related as second cousins or closer $(F \geq 0.0156)$ and their progeny account for an estimated $10 \%$ of the global population [6]. In Peru, the rate of consanguineous marriages is estimated to be between $1 \%$ and $4 \%$ [6].

Numerous studies have applied molecular karyotyping with microarray analysis in identifying subtle DNA changes to explain the causation of unexplained developmental/intellectual disabilities, autism spectrum disorders, and multiple congenital anomalies. Microarrays can identify submicroscopic deletions and duplications not found by routine G-banded chromosome studies. Chromosomal microarray analysis is now considered in the first tier of clinical genetic testing in those patients presenting with the above common reasons for referral [8]. The testing is readily available in most genetic centers in high income or industrialized societies, but yet many patients in underprivileged or low income countries do not have readily available access to these genetic services. Hence, the utility of microarray studies in infants and children characterized with atypical development has not been established in low income or poor countries.

Herein, we report our genetics experience with high resolution microarrays in individuals after 6 months of age from Peru, a low income country where these services are limited, and those at risk for developmental disability, autism, or aberrant behavior. The goals of our study consisted of the following: (1) to identify, screen, enroll, and assess Peruvian infants and children at a young age with atypical development or with behavioral concerns for high resolution microarray analysis to study copy number variation and consanguinity status and to generate clinical case reports and (2) to test whether an adequate DNA yield can be obtained from buccal cells collected with cotton swabs only and stored at room temperature until shipped to the USA for DNA isolation.

\section{Subjects and Methods}

Participants were recruited as part of an innovative national outreach effort to identify and treat children with developmental problems in urban, rural, underprivileged, or otherwise underserved regions of Peru. Participants were solicited by approved television, radio, and newspaper announcements throughout Peru, requesting parents to respond if they had a child between the ages of 6 to 36 months and concerns regarding their child's development, cognition, communication, behavioral problems especially self-injury (SIB), stereotypy and aggression, or a family history of disabilities, medical conditions, or issues related to environmental or other unexplained factors. The parents who responded to the announcements called the Telephone Triage Service at
Centro Ann Sullivan del Peru (CASP), a developmental training center affiliated with the University of Kansas and located in Lima. After brief discussion of their appropriateness for the project, 341 respondents were invited to CASP for a screening interview by trained interviewers/examiners, using the Parental Concerns Questionnaire (PCQ), a checklist based upon risk factors related to behavioral problems among people with developmental disabilities (DD) [9]. If parents responded "yes" to any of these risk factors, they were included in the study. After screening, 262 families were invited to attend the CASP and 233 agreed to undergo cognitive and communication assessments, developmental pediatric and dental exams and visual, auditory, and behavioral evaluations performed by 10 teams of personnel trained and monitored by eight consultants from the USA who were experts in their respective disciplines (summarized by MayoOrtega et al.) [10]. The parents of all participants signed consent forms approved by the Human Subjects Institutional Review Board Committees at CASP and the Human Subjects Committee at the University of Kansas prior to evaluation.

Participants were screened using the Parent Concerns Questionnaire, a 15-item yes/no checklist based on an extensive list of 53 developmental risk factors for severe behavioral problems in children across nine domains [10]. The domains of concern were intellectual disability; communication impairment; genetic syndromes; family history of brain disorders; several medical conditions; psychiatric factors; neurochemical and metabolic factors; neuropsychological factors; and developmental motor factors.

Each participant received (1) anthropometric measures of height, weight, and head circumference; (2) developmental pediatric exam with consults by local Peruvian specialists from pediatric neurology, medical genetics, nutrition, and child psychiatry as needed. If the pediatricians suspected autism, the Child Autism Rating Scale (CARS) was completed by the parents [11]; (3) behavior was assessed using the Behavior Problems Inventory (BPI-01) as the primary instrument [12]; (4) cognitive assessment was undertaken using the Bayley Scales of Infant Development, Third Edition (BSID III) [13]; (5) communication was assessed using the Communication and Symbolic Behavior Scales (CSBS) [14]; (6) visual assessment was performed by a pediatric ophthalmologist from the USA; (7) hearing assessment was performed by a hearing specialist from the USA; and (8) dental assessment was performed by a Peruvian odontologist. The latter three assessments were performed by experts in treating people with DD.

Both the BSID and CSBS are standardized measures with a standard deviation of 15 . Both scales are designed for the age group under study. One standard deviation below the mean of 100 for these instruments is a commonly accepted cut-off considered to be at-risk for developmental delay. Two standard deviations are often defined as intellectual or communication disability along with a measure of social performance ability. A standard cut-off score for autism on the CARS is 35 but used as a screening measure only.

Four cotton swabs that were readily available locally were used to collect buccal cells from each child by the medical examiner as part of a physical examination. Saliva 
collection kits for DNA isolation were not available locally. Parents were not available for specimen collection. Swabs were then stored at room temperature in a dry location for DNA isolation and genetic testing to be performed later in the USA according to protocols reported by Rethmeyer et al. [15]. For this preliminary study, DNA was isolated from 111 samples which represented about one-half of the 233 participants from which the buccal samples were collected. These DNA samples were then monitored for sufficient quantity (e.g., $3 \mu \mathrm{g}$ ) and quality [i.e., intact, nondegraded single DNA band by gel electrophoresis with an acceptable optic density spectroscopic reading (e.g., 1.7)] required for high resolution microarray analysis. Approximately $50 \%$ of the DNA samples obtained did not meet the laboratory criteria required for high resolution microarray hybridization, reflecting the buccal swab collection process, handling, and storage prior to DNA isolation. Participants were excluded from microarray analysis with an inadequate DNA yield or with a known cause of their atypical development and/or aberrant behavior (e.g., Down syndrome, neurofibromatosis, fragile X syndrome, pre- or postnatal trauma, infections, and hydrocephalus or brain malformations) after review of medical, laboratory, and brain imaging records (if available) and clinical assessments with examinations performed by local medical experts and specialists. Of the approximately 50 DNA samples meeting laboratory criteria for microarray analysis, funding was available to analyze 15 representative children (12 males: 3 females, mean age $\pm \mathrm{SD}=28.1 \mathrm{~m} \pm$ $7.9 \mathrm{~m}$; age range $14 \mathrm{~m}-41 \mathrm{~m}$; see Table 1) selected at random from those not having a known cause for their atypical development and/or aberrant behavior.

\section{Results}

3.1. High Resolution Microarray Hybridization and Analysis. Two high resolution microarray platforms widely utilized in the clinical setting for patients in higher income countries were chosen for our study participants from Peru. Five DNA samples were analyzed at the University of Kansas Medical Center Genomics Core Facility using the Affymetrix Genome-wide Human SNP Array 6.0 version utilizing 1.8 million copy number variant $(\mathrm{CNV})$ and single nucleotide polymorphism (SNP) DNA probes. The remaining 10 samples were analyzed by iLife Discoveries (Haryana, India) using the Affymetrix Cytoscan HD Array utilizing 2.3 million copy number variant (CNV) and single nucleotide polymorphism (SNP) DNA probes (Affymetrix, Santa Clara, CA). The Affymetrix Chromosome Analysis Suite 1.2.2 software version was used for determination of the number, location, and size of the CNV (i.e., deletions or duplications) or regions of homozygosity $(\mathrm{ROH})$ by downloading and analyzing the microarray genome-wide SNP and CNV probe data on each participant and following standards recommended by the manufacturer of the high resolution microarrays for determination of genetic lesions and copy number variation. Deletions (losses) and duplications (gains) in the genome were based on the number of DNA probes deleted (or duplicated) over and within an established genomic distance and the number of involved DNA markers. We chose $100 \mathrm{~kb}$ size gains and losses across the genome with a minimum of 50 markers to make a call for a genomic abnormality. For regions of known significance in the human genome, a minimal setting of $25 \mathrm{~kb}$ in size and 50 markers for gains and losses were needed. For ROH, a minimal setting of 3 to $5 \mathrm{Mb}$ was utilized.

In lieu of family history or pedigree analysis, we also estimated consanguinity and the coefficient of inbreeding $(F)$ was calculated based on $\mathrm{ROHs}$ for each participant using the model proposed by Kearney et al. [3]. The coefficient of inbreeding considers regions of homozygosity from autosomal chromosomes greater than $3 \mathrm{Mb}$ in size. Sex chromosomes are excluded due to limited recombination of the $\mathrm{X}$ chromosome. The sum of $\mathrm{ROH}$ greater than $3 \mathrm{Mb}$ from autosomal chromosomes is divided by the total length of autosomal chromosomes in humans (equal to 2,867,733 kb or 2,867.733 Mb in size; using the Human Genome Browserhg 18 assembly-http://www.genome.ucsc.edu/index.html) [16] to estimate the percentage of inheritance by descent or consanguinity relationship. The predicted percentage of genetic material identical by descent for an individual can then be assessed for various degrees of relationship (e.g., $25 \%$ for first degree relatives (parents, children, and siblings) in which one-fourth of the genes are identical by descent with regions of homozygosity determined by lack of DNA polymorphisms $(F=0.25) ; 12.5 \%$ for second degree relatives (grandparents, grandchildren, half-siblings, uncles, aunts, nieces, and nephews) $(F=0.125) ; 6 \%$ for third-degree relatives (first cousins) $(F=0.0625)$ ). Couples related as fifth degree relatives (second cousins or closer) with an $F \geq 0.0156$ are considered consanguineous [6].

3.2. Clinical Presentations. Clinical presentation of the 15 children studied included development delay or aberrant behavior as characterized by interdisciplinary assessments listed in Table 1. Only one child (participant 21) had a normative score for both intelligence and communication ability. Three children (participants 144, 146, and 188) were untestable because of the severity of their intellectual disability. Six children had high CARS scores while the remaining children were not suspected of having autism by the developmental pediatricians.

Assessments for SIB, stereotyped behavior, and aggression were performed with the BPI-01 instrument. Normative data showed that 10 of the 15 children (participants 21, 32, 44, $94,102,120,146,176,218$, and 237) had high scores according to Rojahn et al. [17]. These observations were especially true for both SIB and aggression and much less for stereotyped behavior. The interdisciplinary assessments and their relation to risk factors for behavioral problems used in our study are described elsewhere [9].

The microarray genetic findings of the 15 children with atypical development and autism or aberrant behavior are summarized in Table 2. Participant 21 was a male presenting at 28 months of age with atypical development. He was large for his age and had normal cognition but with increased stereotypical behavior and autistic features. He had a 7q22.3 


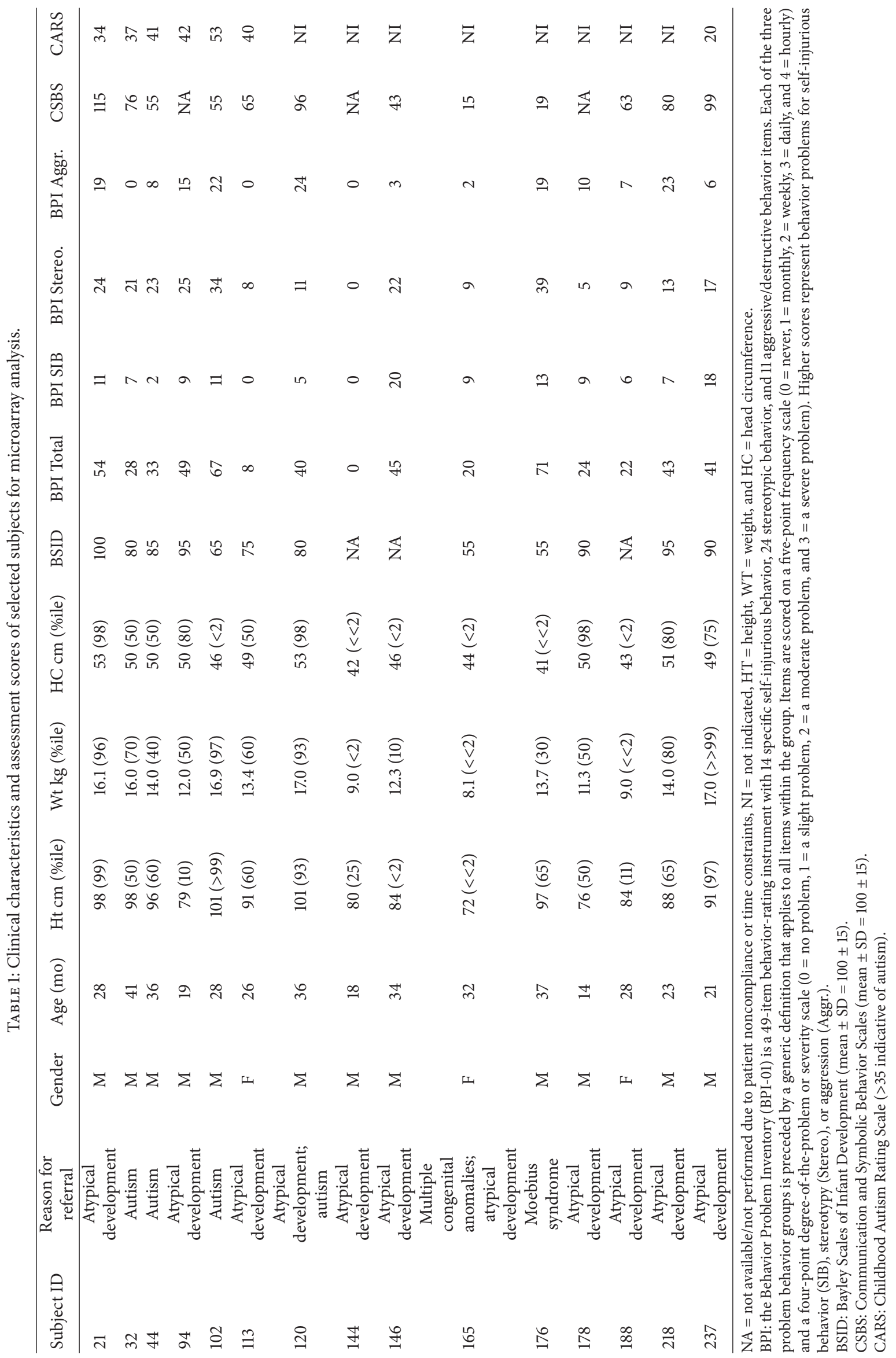




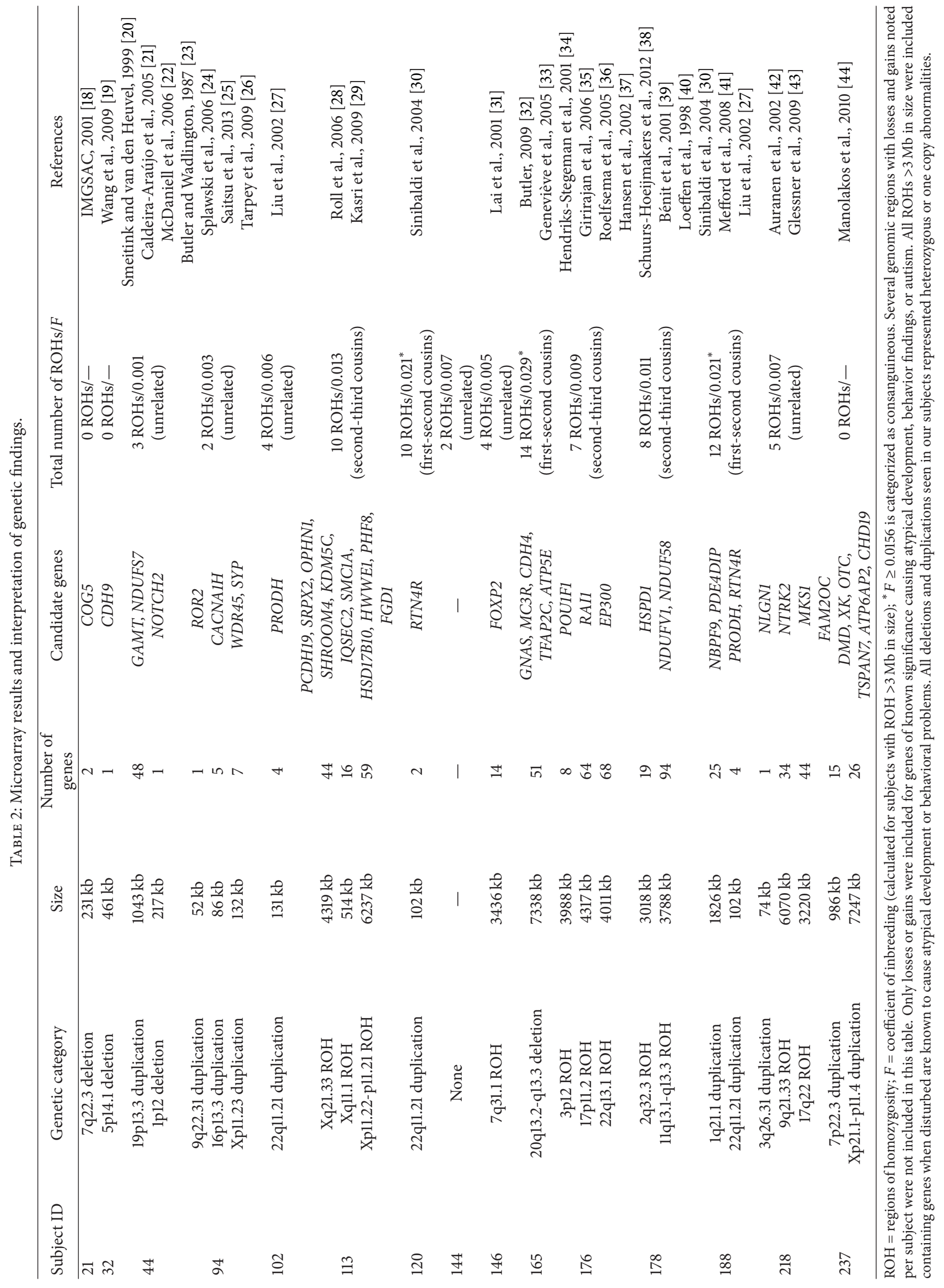


deletion $(231 \mathrm{~kb}$ in size). This small deletion included the COG5 gene which is located in the autism susceptibility 1 (AUTS1) locus known to cause atypical development and autism [18] supporting an underlying cause of his developmental/behavioral problems.

Participants 32 and 44 are both males at 41 months and 36 months of age, respectively, and presented with normal cognition. The CARS and BPI scores supported the diagnosis of autism. Participant 32 had a 5p14.1 deletion including the $\mathrm{CDH} 9$ gene, one of the cadherin gene members reported to play a role in autism and supported by genome-wide association studies indicating this genomic region to be highly associated with autism [19]. Participant 44 showed disturbed NDUFS7 and GAMT genes due to a chromosome $19 \mathrm{p} 13.3$ duplication ( $1043 \mathrm{~kb}$ in size). These genes may cause developmental delay, psychomotor retardation, hypotonia, regression, and metabolic problems [20, 21]. He also had a $217 \mathrm{~kb}$ deletion of chromosome 1p12-p11.2 including the NOTCH2 gene. Notch receptor defects can cause failure to thrive, renal abnormalities, heart defects, and learning disabilities [22] and may have contributed to his clinical findings. The coefficient of inbreeding for participant 44 indicated that the parents were unrelated.

Participant 94 is a 19-month-old male with several microarray findings including a very small 9q22.31 partial duplication ( $52 \mathrm{~kb}$ in size) including the ROR2 gene which encodes a receptor tyrosine kinase when defective causes Robinow syndrome. This syndrome is characterized by short stature, mesomelia, brachydactyly, a particular facial appearance, and developmental delay [23]. He showed high BPI and CARS scores representing behavioral problems and autism but had normal stature and no dysmorphic features. In addition, a very small partial 16p13.3 duplication was found including the CACNA1H gene encoding a calcium ion channel subunit, causing susceptibility to epilepsy [24]. A partial duplication of the WDR45 gene was also found at Xp11.23, which regulates the assembly of multiprotein complexes and protein-protein interactions. Truncating and missense mutations of this gene have been implicated in neurodegeneration with brain iron accumulation leading to intellectual disability, dementia, dystonia, and tremors [25]. In addition, the SYP gene was also duplicated at Xp11.23 and is an integral membrane protein that regulates synaptic vesicle endocytosis associated with X-linked intellectual disability and epilepsy [26]. The coefficient of inbreeding indicates that the parents were unrelated.

Participant 102 is a 28 -month-old male with tall stature and microcephaly with a very small gain of the $22 q 11.21$ region containing the $P R O D H$ gene encoding proline dehydrogenase. This enzyme catalyzes the conversion of proline to pyrroline-5-carboxylate with disturbances linked to the susceptibility of schizophrenia, autism, hyperactivity, aggression, and intellectual disability [27]. He had BSID, BPI, CSBS, and CARS scores indicative of developmental delay, behavioral and communication problems, and autism. The coefficient of inbreeding indicated that the parents were unrelated.

Participant 113 is a 26-month-old female with an $\mathrm{ROH}$ at Xq21.33-q22.1 including the PCDH19 and SRPX2 genes.
PCDH19 encodes protocadherin 19 known to play a role in autism while the SRPX2 gene is associated with Rolandic epilepsy, intellectual disability, and speech dyspraxia [28]. She had CSBS and CARS scores indicating communication problems and autism. Additional ROHs at Xq11.1-q12 and Xp11.22-p11.21 included genes (e.g., OPHN1) when defective are implicated in the development of intellectual disabilities. The OPHN1 geneencodes the Rho GTPase-activating protein associated with X-linked intellectual disabilities and cerebellar hypoplasia [29]. The coefficient of inbreeding represented a second-third cousin relationship.

Participant 120 is a 36 -month-old male who showed a very small gain at 22q11.21 which contains the RTN4R gene for susceptibility to schizophrenia by encoding a protein component of myelin and preventing axonal regeneration [30]. His BPI scores indicated a high level of aggression. The coefficient of inbreeding represented a first-second cousin relationship, indicating consanguinity.

Participant 146 is a 34 -month-old male with growth retardation and showed an $\mathrm{ROH}$ identified at 7q31.1-q31.2 which contains the FOXP2 gene encoding forkhead box P2, a putative transcription factor and DNA binding domain. Mutations leading to haploinsufficiency of this gene can result in speech-language disorder-1 [31]. His CSBS score indicated impaired communication and symbolic behavior skills. The coefficient of inbreeding indicated that the parents were unrelated.

Participant 165 is a 32 -month-old female with a 20q13.2q13.3 deletion (7,339 kb in size) involving GNAS, MC3R, CDH6, TFAP2C, and approximately 50 other genes. GNAS beinga complex locus containing four genes with three of the genes (XLAS, NESP, and NESPAS) being imprinted or expressed differently depending on the parent of origin (reviewed by Butler [32]). The MC3R (melanocortin 3 receptor) gene is known to play a role in obesity while the $\mathrm{CDH} 4$ is a member of the cadherin gene family mediating calciumdependent cell-cell adhesion and plays a role in autism. TFAP2C modulates the transcriptional activity of vitamin A and target genes via the retinoic receptors and is expressed in migrating neural crest cells in the developing embryo [33]. She presented with multiple congenital anomalies, generalized growth retardation, developmental delay, and impaired communication and symbolic behavior skills. The coefficient of inbreeding represented a first-second cousin relationship, indicating consanguinity.

Participant 176 is a 37-month-old male with ROHs noted on chromosomes 3, 17, and 22 within regions where several genes (i.e., POU1F1, RAI1, and EP300) associated with intellectual disability are found. If mutated and inherited in a recessive pattern then intellectual disability, hypotonia, speech delay, self-injury, microcephaly and behavioral problems can occur [34-36]. Several of these features were seen in this participant including Moebius syndrome, microcephaly, developmental delay, stereotypy, and impaired communication and symbolic behavior skills. The coefficient of inbreeding represented a second-third cousin relationship.

Participant 178 is a 14-month-old male with macrocephaly and two ROHs on chromosomes 2 and 11 involving the HSPD1, PACS1, NDUFV1, and NDUFS8 genes that may 
play a role in psychomotor delay, hypotonia, and atypical development [37-40]. He did present with atypical development. The coefficient of inbreeding represented a secondthird cousin relationship.

Participant 188 is a 28 -month-old female who was small for age with microcephaly and showed a 1q21.1 duplication $(1,826 \mathrm{~kb}$ in size) which included 25 genes. Duplications of this chromosome region have been reported previously in individuals with mild to moderate global delay, abnormal head size, autism, self-injury, stereotypy, speech delay, and mild facial dysmorphism [41]. In addition, this participant also had a very small 22q11.21 duplication including the $P R O D H$ and RTN4R genes, as stated previously both reportedly play a role in schizophrenia [27, 30]. She presented with atypical development and impaired communication and symbolic behavior skills. The coefficient of inbreeding represented a first-second cousin relationship, indicating consanguinity.

Participant 218 is a 23 -month-old male who showed a very small gain at 3q26.31 representing a partial duplication of the NLGN1 gene which encodes a neuroligin. Neuroligins function as ligands for neurexins or cell-surface receptors. The neurexin/neuroligin complex is $\mathrm{Ca}(2+)$-dependent and present at synapses in the central nervous system and required for efficient neurotransmission involved in the formation of synaptic contacts proposed to cause autism [42, 43]. In addition, ROHs at 9q21.33-q22.2 and 17q22-q23.2 were found and contained genes (NTRK2, MKS1) that may play a role in obesity and developmental or speech delay $[45,46]$. He presented with atypical development. The coefficient of inbreeding indicates that the parents were unrelated.

Participant 237 is a 21-month-old male who was large for age and showed a gain at 7p22.3 which includes the FAM20C gene associated with Raine syndrome. This syndrome is characterized by short stature, microcephaly, craniofacial dysplasia, renal abnormalities, developmental delay, and selfstimulating behavior [47]. In addition, gains of genetic information on the $\mathrm{X}$ chromosome seen in the participant included several genes involved with muscle development and learning problems. These disturbances could explain the atypical development and behavior problems seen at an early age in our participant although other features of Raine syndrome were not present.

\section{Discussion}

Chromosomal microarray analysis is now considered in the first tier of clinical genetic testing when evaluating children presenting with unexplained developmental delay/intellectual disabilities, autism spectrum disorders, and/or multiple congenital anomalies. This method yields a much higher result than routine chromosome studies by detecting small submicroscopic deletions and duplications in the genome. Our study is the first to undertake advanced genetic testing using high resolution microarray technology at such a young age (i.e., in infants) systematically ascertained and assessed for atypical development and aberrant behavior from an underserved population in a low income country (Peru).
We utilized locally available cotton swabs as salvia collection kits with DNA preservative were not accessible to collect buccal cells for later use in microarray hybridization. DNA collected from approximately one-half of the buccal cell samples was acceptable for microarray analysis indicating an overall suboptimal yield. Several previous studies using chromosomal microarrays have shown a diagnostic yield of $11 \%$ to $20 \%$ with clinically significant CNVs in those individuals presenting with unexplained intellectual disabilities in high income countries (e.g., in the USA) [2], while a yield of $16 \%$ was found in a cohort of Greek children with developmental delay [44].

Detection of regions of homozygosity ( $\mathrm{ROH}$ ) with microarray technology in itself is not diagnostic for an underlying condition and may have no clinical outcome. However, when present in multiple areas throughout the genome versus long stretches of homozygosity confined to a single chromosome, then these regions may be due to identical by descent or from a common (or shared) ancestor raising the possibility that mutant recessive gene alleles if present in these regions could lead to genetic disorders. If these regions are large (e.g., $>3 \mathrm{Mb}$ ), then the likelihood increases for inheriting a genetic disorder with clinical consequences [3]. ROHs less than $10 \mathrm{Mb}$ are relatively common and likely result from a common ancestor but should not be overlooked when examining for genes in these regions causing atypical development and/or aberrant behavior impacting neurological development or function.

When parents of a child share a recent common ancestor then this relationship becomes consanguineous in nature with the closer relationship having a greater likelihood in producing a child with two copies of a deleterious recessive gene allele mutation received from the parents. Conversely, if one or more regions show extended homozygosity particularly longer than $10 \mathrm{Mb}$ in size on a single chromosome, then the most likely cause is uniparental disomy with loss of heterozygosity of the entire chromosome (error in meiosis II) or as the segmental type (reviewed by Papenhausen et al. [4]). This event is supported by SNP probe data without evidence of polymorphisms within the involved regions and with $\mathrm{CNV}$ data showing a normal or nondeletion status for the specific chromosome region or conversely the entire chromosome.

Unfortunately, no data were available from the parents. The detection of high levels of $\mathrm{ROH}$ without parental data limits accurate interpretation for consanguinity and one can only conclude that consanguinity is present in the community in which the participant lives. Any follow-up study should include parents and demographic survey with questions on family structure/consanguinity and socioeconomic status.

Of the 15 children selected at random to study with microarray analysis, 10 had genomic duplications or deletions containing genes when defective are known to be associated with neurodevelopment and function including autism, behavior problems, schizophrenia, and developmental delay. Therefore, these rearrangements harboring genes become candidates for contributing to the clinical presentations observed in our participants. Two participants had identifiable microduplication/deletion syndromes including participant 165 with a 20q13.2-q13.33 deletion encompassing 
the complex GNAS locus and other genes within the region (see Table 2). This deletion has previously been reported in conjunction with intellectual disability, severe pre- and postnatal growth retardation, facial dysmorphism, mild psychomotor retardation, and hypotonia [33]. Participant 188 was identified as having a 1q21.1 duplication, repeatedly identified in patients with autism and/or intellectual disability. Hence, four of the 15 children had a deletion involving four different chromosomes with deletions ranging from a small $217 \mathrm{~kb}$ to a large 7,338 kb size. Seven of the 15 children were identified with 11 duplications involving eight different chromosomes and the duplications ranged from a very small $52 \mathrm{~kb}$ to a large $7,247 \mathrm{~kb}$ size. The deletions containing genes identified in participants 21,32, 44, and 165 likely accounted for their clinical presentations and similarly with chromosomal duplications found in seven of the children (participants 44, 94,102,120,188, 218, and 237). Very small duplications or deletions in the genome particularly those $<200 \mathrm{~kb}$ in size should be considered preliminary without further characterization of the genes involved in the respective disturbed genomic regions. Whether the reported findings would impact the phenotype or likely be pathogenic would require more testing which is beyond the scope of this study.

Five of the children (participants 113, 146, 176, 178, and 218) in our study had ROHs which may have contributed to their clinical presentation if disturbed recessive candidate gene alleles were present in the extended stretches of homozygosity and thus these participants would have received two copies. The ROHs ranged from 3,018 to 6,237 kb in size. Three children did not have sufficient SNP data for calculation of the coefficient of inbreeding to determine inbreeding status (no ROHs seen). Six participants were found to be unrelated while three showed ROHs consistent with a first-second cousin relationship (consanguineous) but had recognized chromosomal structural anomalies as well including genes associated with developmental and/or behavioral problems. Three participants showed a second-third cousin relationship but limitations in our study prevented detailed family history and parental physical examinations with collection of specimens for parental laboratory evaluations in order to analyze CNVs or SNPs for determining familial versus de novo CNV status or consanguinity. Limitations in our study included lack of resources and access of parental data and laboratory evaluations to analyze CNVs.

In summary, our study showed that in less developed countries high resolution microarray analysis can be done where blood collection and specimen processing may be limited using locally available cotton swabs for buccal cell collection without a DNA preservative and storage at room temperature. However, as expected, the capacity to provide adequate quantity and quality of DNA was suboptimal with about one-half of the specimens yielding DNA suitable for high resolution microarray analysis. Saliva is now becoming a more common specimen source for collection of DNA for genetic testing particularly for the pediatric age group and the use of a DNA preservative. Two-thirds of our participants showed DNA copy number variation consistent with deletions or duplications in genomic regions containing known or candidate genes for atypical development or behavioral problems. One-fifth of the study participants showed evidence of consanguinity but parental samples were not available. We conclude that advanced genetic testing can yield meaningful data even in suboptimal sample collection conditions with limited resources.

\section{Conflict of Interests}

The authors associated with the University of Kansas declare that there is no conflict of interests regarding the publication of this paper.

\section{Acknowledgments}

The authors would like to thank the families who participated in this study as well as professionals and staff at CASP, Lima, Peru. This study would not have been successful without their involvement. This study was partially supported by the NIH Fogarty International Research (HD060500), NICHD HD02528 and the Angelman, Rett and Prader-Will Syndrome Consortium (U54 HD06122) as a part of the National Institutes of Health (NIH) Rare Disease Clinical Research Network (RDCRN) supported through collaboration between the NIH Office of Rare Diseases Research (ORDR) at the National Center of Advancing Translational Science (NCATS), and the National Institute of Child Health and Human Development (NICHD). The content is solely the responsibility of the authors and does not necessarily represent the official views of the National Institutes of Health.

\section{References}

[1] M. G. Butler, E. L. Youngs, J. L. Roberts, and J. A. Hellings, "Assessment and treatment in autism spectrum disorders: a focus on genetics and psychiatry," Autism Research and Treatment, vol. 2012, Article ID 242537, 11 pages, 2012.

[2] J. L. Roberts, K. Hovanes, M. Dasouki, A. M. Manzardo, and M. G. Butler, "Chromosomal microarray analysis of consecutive individuals with autism spectrum disorders or learning disability presenting for genetic services," Gene, vol. 535, no. 1, pp. 7078, 2014.

[3] H. M. Kearney, J. B. Kearney, and L. K. Conlin, "Diagnostic implications of excessive homozygosity detected by SNP-Based microarrays: consanguinity, uniparental disomy, and recessive single-gene mutations," Clinics in Laboratory Medicine, vol. 31, no. 4, pp. 595-613, 2011.

[4] P. Papenhausen, S. Schwartz, H. Risheg et al., "UPD detection using homozygosity profiling with a SNP genotyping microarray," American Journal of Medical Genetics Part A, vol. 155, no. 4, pp. 757-768, 2011.

[5] M. G. Butler, "Prader-Willi syndrome: obesity due to genomic imprinting," Current Genomics, vol. 12, no. 3, pp. 204-215, 2011.

[6] A. H. Bittles and M. L. Black, "Consanguinity, human evolution, and complex diseases," Proceedings of the National Academy of Sciences of the United States of America, vol. 107, no. 1, pp. 17791786, 2010. 
[7] E. M. Morrow, S.-Y. Yoo, S. W. Flavell et al., "Identifying autism loci and genes by tracing recent shared ancestry," Science, vol. 321, no. 5886, pp. 218-223, 2008.

[8] G. B. Schaefer and N. J. Mendelsohn, "Clinical genetics evaluation in identifying the etiology of autism spectrum disorders: 2013 guideline revisions," Genetics in Medicine, vol. 15, no. 5, pp. 399-407, 2013.

[9] S. R. Schroeder, J. Marquis, R. M. Reese et al., "Risk factors for self-injury, aggression, and stereotyped behavior among infants and toddlers at risk for intellectual and developmental disabilities," American Journal on Intellectual and Developmental Disabilities, vol. 119, no. 4, pp. 351-370, 2014.

[10] L. Mayo-Ortega, R. Oyama-Ganiko, J. Leblanc et al., "Mass screening for severe problem behavior among infants and toddlers in Peru," Journal of Mental Health Research in Intellectual Disabilities, vol. 5, no. 3-4, pp. 246-259, 2012.

[11] E. Schopler, R. Reichler, and B. Renner, The Childhood Autism Rating Scale (CARS), Western Psychological Services, Los Angeles, Calif, USA, 1988.

[12] J. Rojahn, J. L. Matson, D. Lott, A. J. Esbensen, and Y. Smalls, "The behavior problems inventory: an instrument for the assessment of self-injury, stereotyped behavior, and aggression/destruction in individuals with developmental disabilities," Journal of Autism and Developmental Disorders, vol. 31, no. 6, pp. 577-588, 2001.

[13] N. Bayley, Manual of the Bayley Scales of Infant and Toddler Development, NCS Pearson, San Antonio, Tex, USA, 3rd edition, 2006.

[14] A. M. Wetherby and B. M. Prizant, Communication and Symbolic Behavior Scales Developmental Profile-1st normed, Paul H. Brookes Publishing, Baltimore, Md, USA, 2002.

[15] J. A. Rethmeyer, X. Tan, A. Manzardo, S. R. Schroeder, and M. G. Butler, "Comparison of biological specimens and DNA collection methods for PCR amplification and microarray analysis," Clinical Chemistry and Laboratory Medicine, vol. 51, no. 5, pp. e79-e83, 2013.

[16] Human Genome Browser-hg 18 assembly, 2014, http://www.genome.ucsc.edu/index.html.

[17] J. Rojahn, E. W. Rowe, A. C. Sharber et al., "The behavior problems inventory-short form for individuals with intellectual disabilities: part I: development and provisional clinical reference data," Journal of Intellectual Disability Research, vol. 56, no. 5, pp. 527-545, 2012.

[18] International Molecular Genetic Study of Autism Consortium (IMGSAC), "A genomewide screen for autism: strong evidence for linkage to chromosomes 2q, 7q, and 16p," The American Journal of Human Genetics, vol. 69, no. 3, pp. 570-581, 2001.

[19] K. Wang, H. Zhang, D. Ma et al., "Common genetic variants on 5 p14.1 associate with autism spectrum disorders," Nature, vol. 459, no. 7246, pp. 528-533, 2009.

[20] J. Smeitink and L. van den Heuvel, "Human mitochondrial complex I in health and disease," The American Journal of Human Genetics, vol. 64, no. 6, pp. 1505-1510, 1999.

[21] H. Caldeira-Araújo, W. Smit, N. M. Verhoeven et al., "Guanidinoacetate methyltransferase deficiency identified in adults and a child with mental retardation," American Journal of Medical Genetics, vol. 133, no. 2, pp. 122-127, 2005.

[22] R. McDaniell, D. M. Warthen, P. A. Sanchez-Lara et al., "NOTCH2 mutations cause Alagille syndrome, a heterogeneous disorder of the notch signaling pathway," The American Journal of Human Genetics, vol. 79, no. 1, pp. 169-173, 2006.
[23] M. G. Butler and W. B. Wadlington, "Robinow syndrome: report of two patients and review of literature," Clinical Genetics, vol. 31, no. 2, pp. 77-85, 1987.

[24] I. Splawski, D. S. Yoo, S. C. Stotz, A. Cherry, D. E. Clapham, and M. T. Keating, "CACNA1H mutations in autism spectrum disorders," The Journal of Biological Chemistry, vol. 281, no. 31, pp. 22085-22091, 2006.

[25] H. Saitsu, T. Nishimura, K. Muramatsu et al., "De novo mutations in the autophagy gene WDR45 cause static encephalopathy of childhood with neurodegeneration in adulthood," Nature Genetics, vol. 45, no. 4, pp. 445-449, 2013.

[26] P. S. Tarpey, R. Smith, E. Pleasance et al., "A systematic, largescale resequencing screen of X-chromosome coding exons in mental retardation," Nature Genetics, vol. 41, no. 5, pp. 535-543, 2009.

[27] H. Liu, S. C. Heath, C. Sobin et al., "Genetic variation at the 22q11 PRODH2/DGCR6 locus presents an unusual pattern and increases susceptibility to schizophrenia," Proceedings of the National Academy of Sciences of the United States of America, vol. 99, no. 6, pp. 3717-3722, 2002.

[28] P. Roll, G. Rudolf, S. Pereira et al., "SRPX2 mutations in disorders of language cortex and cognition," Human Molecular Genetics, vol. 15, no. 7, pp. 1195-1207, 2006.

[29] N. N. Kasri, A. Nakano-Kobayashi, R. Malinow, B. Li, and L. Van Aelst, "The Rho-linked mental retardation protein oligophrenin-1 controls synapse maturation and plasticity by stabilizing AMPA receptors," Genes and Development, vol. 23, no. 11, pp. 1289-1302, 2009.

[30] L. Sinibaldi, A. De Luca, E. Bellacchio et al., "Mutations of the Nogo-66 receptor (RTN4R) gene in schizophrenia," Human Mutation, vol. 24, no. 6, pp. 534-535, 2004.

[31] C. S. L. Lai, S. E. Fisher, J. A. Hurst, F. Vargha-Khadem, and A. P. Monaco, "A forkhead-domain gene is mutated in a severe speech and language disorder," Nature, vol. 413, no. 6855, pp. 519-523, 2001.

[32] M. G. Butler, "Genomic imprinting disorders in humans: a mini-review," Journal of Assisted Reproduction and Genetics, vol. 26, no. 9-10, pp. 477-486, 2009.

[33] D. Geneviève, D. Sanlaville, L. Faivre et al., "Paternal deletion of the GNAS imprinted locus (including Gnasxl) in two girls presenting with severe pre- and post-natal growth retardation and intractable feeding difficulties," European Journal of Human Genetics, vol. 13, no. 9, pp. 1033-1039, 2005.

[34] B. I. Hendriks-Stegeman, K. D. Augustijn, B. Bakker, P. Holthuizen, P. C. van der Vliet, and M. Jansen, "Combined pituitary hormone deficiency caused by compound heterozygosity for two novel mutations in the POU domain of the PIT1/POU1F1 gene," Journal of Clinical Endocrinology and Metabolism, vol. 86, no. 4, pp. 1545-1550, 2001.

[35] S. Girirajan, C. N. Vlangos, B. B. Szomju et al., "Genotypephenotype correlation in Smith-Magenis syndrome: evidence that multiple genes in $17 \mathrm{p} 11.2$ contribute to the clinical spectrum," Genetics in Medicine, vol. 8, no. 7, pp. 417-427, 2006.

[36] J. H. Roelfsema, S. J. White, Y. Ariyürek et al., "Genetic heterogeneity in Rubinstein-Taybi syndrome: mutations in both the CBP and EP300 genes cause disease," American Journal of Human Genetics, vol. 76, no. 4, pp. 572-580, 2005.

[37] J. J. Hansen, A. Dürr, I. Cournu-Rebeix et al., "Hereditary spastic paraplegia SPG13 is associated with a mutation in the gene encoding the mitochondrial chaperonin Hsp60," The American Journal of Human Genetics, vol. 70, no. 5, pp. 13281332, 2002. 
[38] J. H. M. Schuurs-Hoeijmakers, E. C. Oh, L. E. Vissers et al., "Recurrent de novo mutations in PACS1 cause defective cranialneural-crest migration and define a recognizable intellectualdisability syndrome," The American Journal of Human Genetics, vol. 91, no. 6, pp. 1122-1127, 2012.

[39] P. Bénit, D. Chretien, N. Kadhom et al., "Large-scale deletion and point mutations of the nuclear NDUFV1 and NDUFS1 genes in mitochondrial complex I deficiency," The American Journal of Human Genetics, vol. 68, no. 6, pp. 1344-1352, 2001.

[40] J. Loeffen, J. A. M. Smeitink, R. Triepels et al., "The first nuclearencoded complex I mutation in a patient with Leigh syndrome," American Journal of Human Genetics, vol. 63, no. 6, pp. 15981608, 1998.

[41] H. Mefford, A. Sharp, C. Baker et al., "Recurrent rearrangements of chromosome 1q21.1 and variable pediatric phenotypes," The New England Journal of Medicine, vol. 359, no. 16, pp. 1685-1699, 2008.

[42] M. Auranen, R. Vanhala, T. Varilo et al., "A genomewide screen for autism-spectrum disorders: evidence for a major susceptibility locus on chromosome 3q25-27," The American Journal of Human Genetics, vol. 71, no. 4, pp. 777-790, 2002.

[43] J. T. Glessner, K. Wang, G. Cai et al., "Autism genome-wide copy number variation reveals ubiquitin and neuronal genes," Nature, vol. 459, pp. 569-573, 2009.

[44] E. Manolakos, A. Vetro, K. Kefalas et al., "The use of arrayCGH in a cohort of Greek children with developmental delay," Molecular Cytogenetics, vol. 3, no. 1, article 22, 2010.

[45] G. S. H. Yeo, C.-C. C. Hung, J. Rochford et al., "A de novo mutation affecting human TrkB associated with severe obesity and developmental delay," Nature Neuroscience, vol. 7, no. 11, pp. 1187-1189, 2004.

[46] C. C. Leitch, N. A. Zaghloul, E. E. Davis et al., "Hypomorphic mutations in syndromic encephalocele genes are associated with Bardet-Biedl syndrome," Nature Genetics, vol. 40, pp. 443448, 2008.

[47] M. A. Simpson, A. Scheuerle, J. Hurst, M. A. Patton, C. Stewart, and A. H. Crosby, "Mutations in FAM20C also identified in non-lethal osteosclerotic bone dysplasia," Clinical Genetics, vol. 75 , no. 3, pp. 271-276, 2009. 

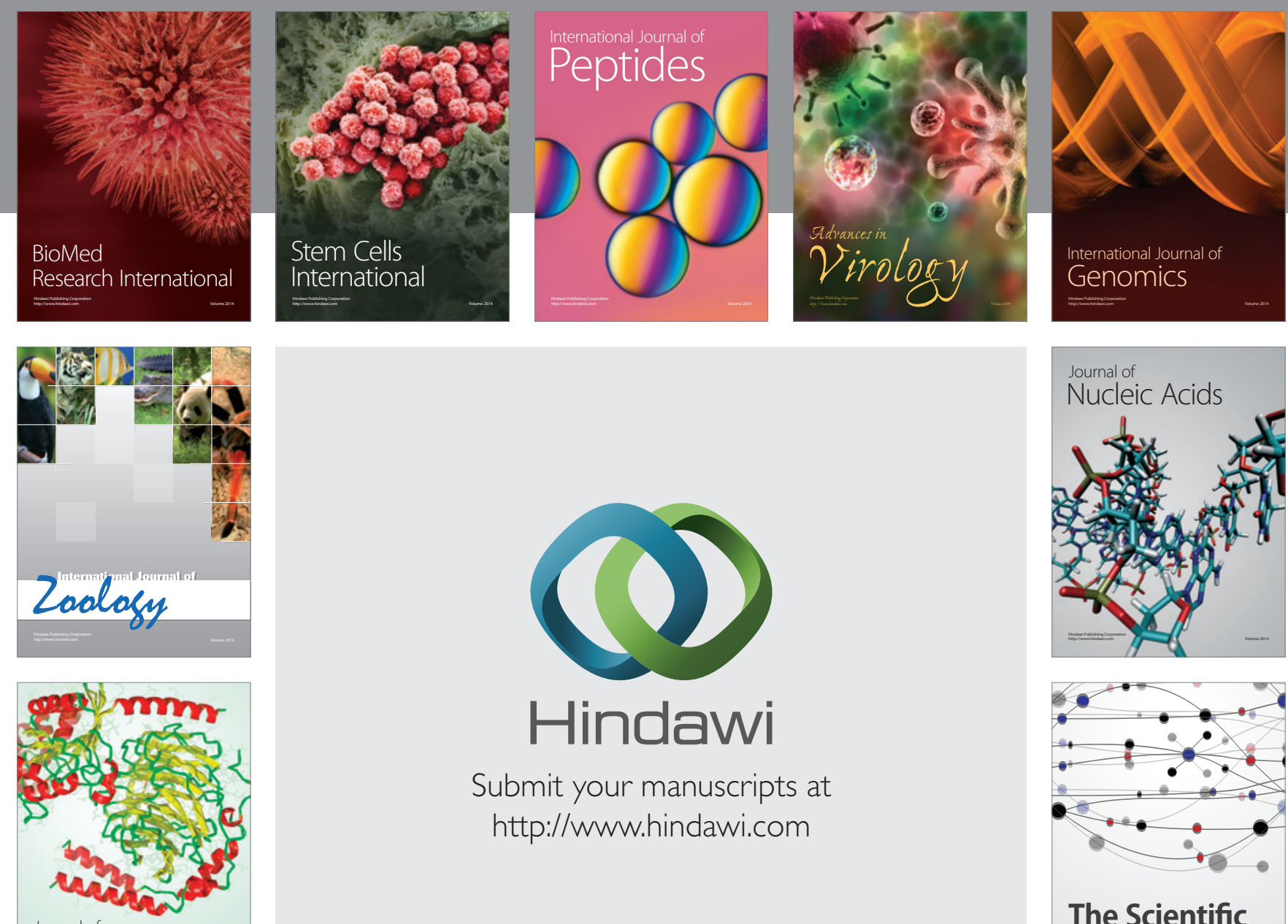

Submit your manuscripts at

http://www.hindawi.com

Journal of
Signal Transduction
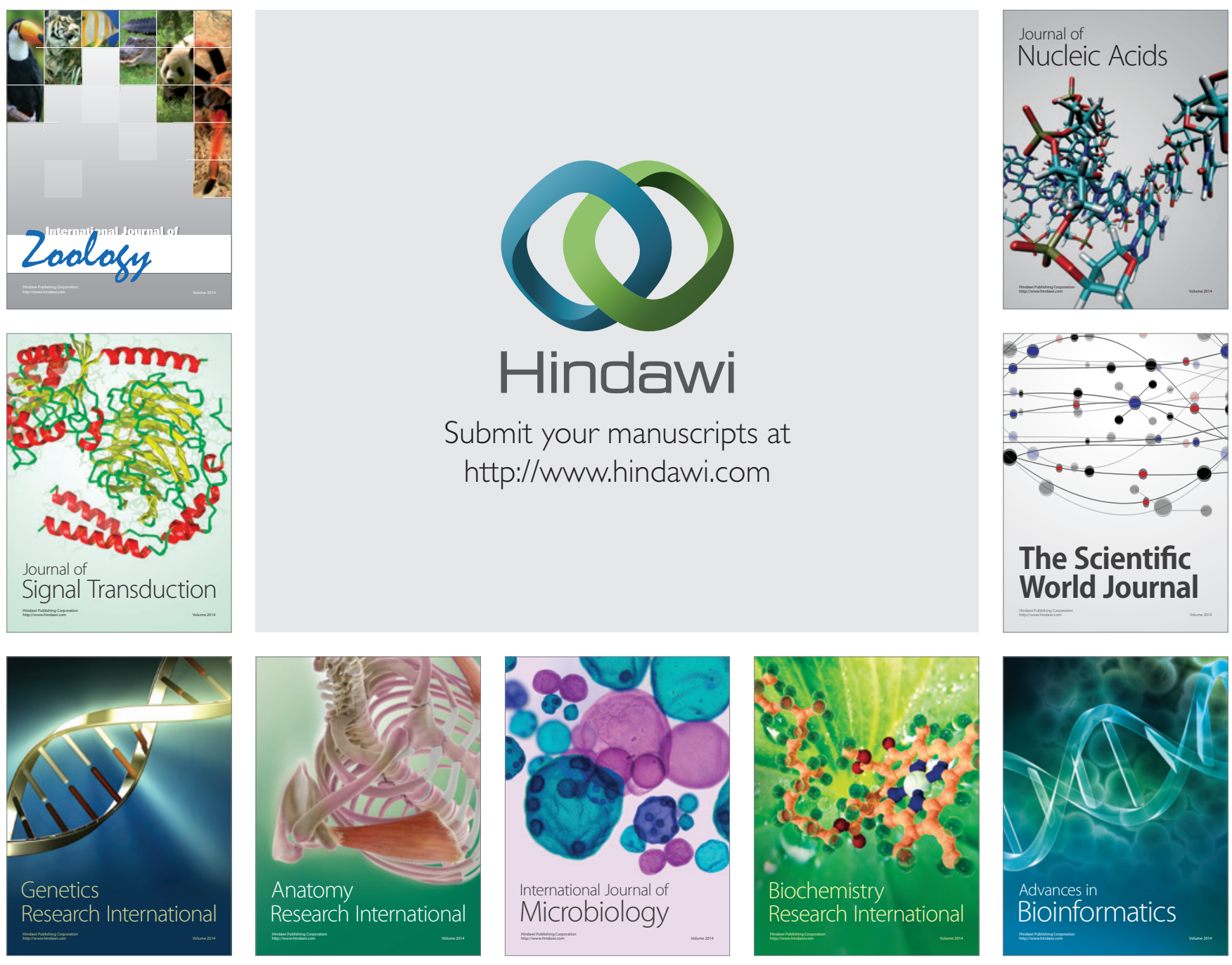

The Scientific World Journal
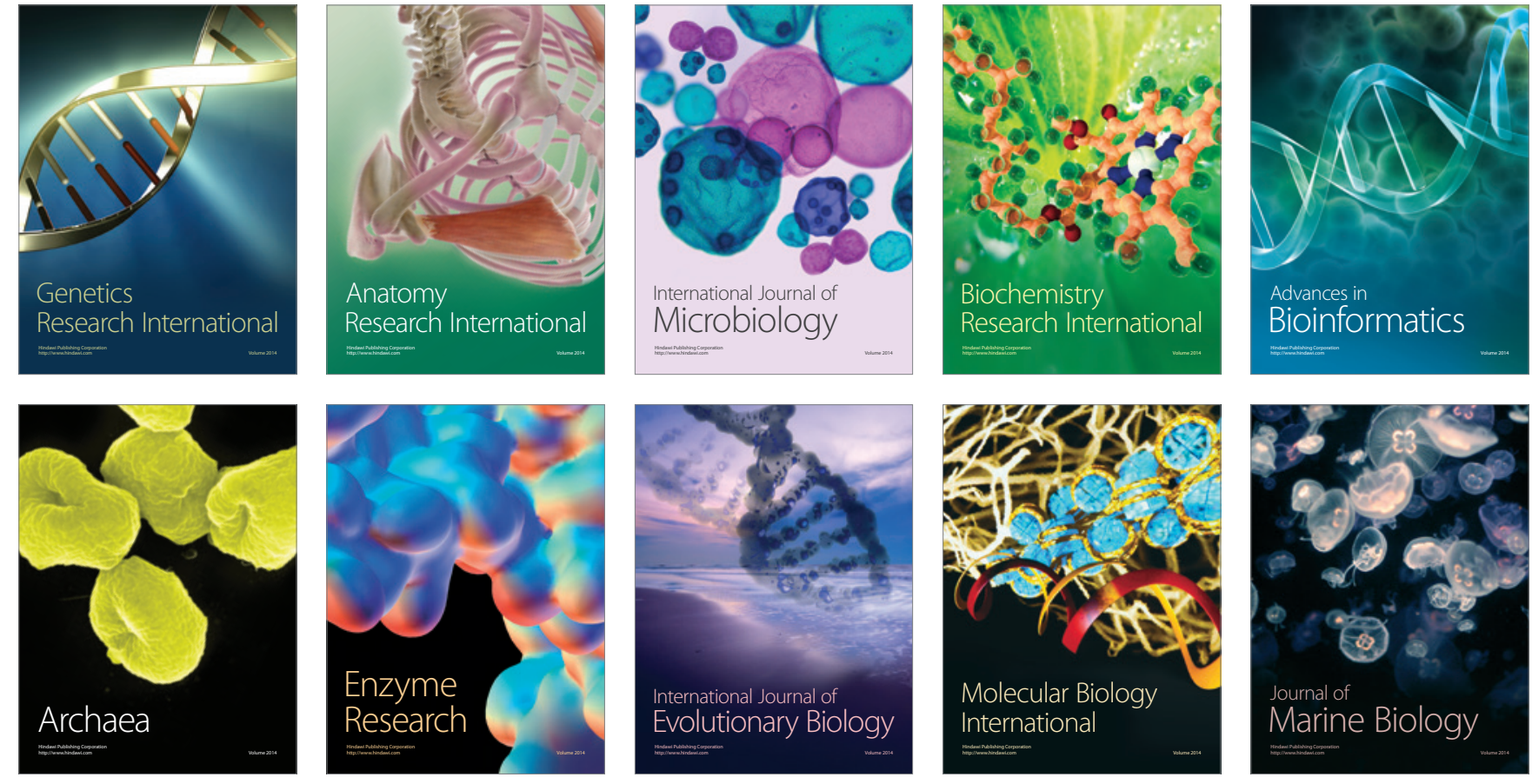